\title{
Induction of Changes in Internal Gas Pressure of Bulky Plant Organs by Temperature Gradients
}

\author{
Kenneth A. Corey and Zhi-Yl Tan \\ Department of Plant and Soil Sciences, University of Massachusetts, Amherst, MA 01003 \\ Additional index words. tomato, pepper, mass flow, transport, apple, carrot, witloof chicory, rhubarb, pokeweed, \\ Phytolacca americana
}

\begin{abstract}
Water manometers were connected to fruits of tomato (Lycopersicon esculentum Mill.) and pepper (Capsicum annuum $\mathrm{L}_{\text {.) }}$, and then fruits were submerged in water baths providing initial temperature gradients between fruit and water of 0 to 19C. Apple (Malus domestics Borkh.) fruits, carrot (Daucus carota L.) roots, witloof chicory (Cichorium intybus L.) roots, rhubarb Rheum rhabarbarum L.) petioles, and pokeweed (Phytolacca americana L.) stems were subjected to water bath temperature gradients of 5C. Internal partial vacuums developed in all organs within minutes of imposing the gradients. The maximum partial vacuums in tomato and pepper fruits increased with increasing temperature gradients. Uptake of water accompanied changes in internal pressure reaching maxima of $17 \%(\mathrm{w} / \mathrm{w})$ and $2 \%(\mathrm{w} / \mathrm{w})$ of pepper and tomato fruits, respectively, after 22 hours. Maximum pressure changes achieved in bulky organs deviated from those predicted by the ideal gas law, possibly due to concomitant changes in gas pressure upon replacement of intercellular spaces with water and dissolution of $\mathrm{CO}_{2}$. Partial vacuums also developed in pepper fruits, rhubarb petioles, and pokeweed stems following exposure to air 15C cooler than initial organ temperatures. Results point to the role of temperature gradients in the transport of liquids and gases in plant organs.
\end{abstract}

The primary mechanism for exchange of metabolic gases between the interior and exterior of bulky plant organs is diffusion (Ben-Yehoshua et al., 1985; Burg and Burg, 1965; Cameron and Yang, 1982; Solomos, 1987). Partial pressure gradients between the interior and exterior of plant organs may occur for individual gases, but the total internal pressure of gases approximates atmospheric pressure for organs in a gaseous environment. The absence of total gas pressure gradients in bulky organs may hold true for organs where gas transport pathlengths, tissue permeabilities, and frequencies of openings on the surface of the organ enable fairly rapid transfer and equilibration of gases (resulting from partial pressure gradients). A large proportion of the surface area of fruits such as tomato and pepper have greatly restricted permeability to gases. For example, the stem scar region of tomato fruit, where diffusion is not restricted greatly, is the site for $97 \%$ of the gas exchange (Cameron and Yang, 1982).

Restricted surface area or additional diffusion barriers for gas exchange, such as water baths for postharvest washing treatments, may permit development of total gas pressure gradients between the organ and its external environment. Roots have solid and aqueous barriers to transport of gases that may limit the rate of equilibration of gases by diffusion.

We suggest that the occurrence of total gas pressure gradients in plant organs maybe a common phenomenon and would serve as the driving force for the transport, via mass flow, of solutions and gases. Specifically, we postulate that temperature gradients, whether natural or imposed, cause the development of total gas pressure gradients in plant tissues. Temperature gradient-induced gas pressure gradients may be an important mechanism for mass flow of solutions and gases in plant tissues and organs. Therefore, the objectives of this study were to determine if changes in gas pressure can be induced by imposing temperature gradients and, if so, to determine the magnitude and extent of the changes.

Received for publication 27 Mar. 1989. Paper no. 2921 of the Massachusetts Agricultural Experiment Station. The cost of publishing this paper was defrayed in part by the payment of page charges. Under postal regulations, this paper therefore must be hereby marked advertisement solely to indicate this fact.

\section{Materials and Methods}

Plant material. Green bell pepper fruits, mature green tomato ('Empire') fruits, apple ('Red Delicious') fruits, endive ('Fare') roots, carrot roots, rhubarb plants, and pokeweed plants were obtained from local farms, storage facilities, and grocery stores. In all experiments, organs selected were free from obvious physical damage, disease, and shape deformities.

Temperature treatments. Plant organs were thermally equilibrated with the air temperature of the laboratory, which ranged from 22 to $25 \mathrm{C}$, depending on the experiment. The $\mathrm{pH}$ of the water was lowered to minimize possible effects of $\mathrm{CO}_{2}$ dissolution on internal pressure changes. Individual organs were submerged in water baths adjusted to $\mathrm{pH} 2.5$ with $10 \%$ (v/v) $\mathrm{HCl}$. Water baths were equilibrated thermally to provide initial temperature gradients of 0 to $19 \mathrm{C}$ between the organ and the water, depending on the experiment. For experiments involving placement of pepper fruits into water warmer than the fruits, the fruits were first cooled to $17 \mathrm{C}$, which was $8 \mathrm{C}$ colder than the laboratory temperature.

Pepper and apple fruits, pokeweed and rhubarb stems, and endive roots preequilibrated at the laboratory temperature were transferred to a cold storage room at $10 \mathrm{C}$ (15C cooler than the laboratory temperature) to determine if pressure gradients developed in the absence of an aqueous barrier.

Pressure measurements. Prior to submergence of bulky organs in water baths, hubless 3.8-cm-long, 18-gauge stainless steel hypodermic needles were inserted through $0.3-\mathrm{cm}$-thick $\times$ 1.3-cm-diameter rubber septa and attached to the surface of pepper and tomato fruits with contact cement (Redy-Mastic, formula 8160, Gibson Homans Co., Twinsburg, Ohio). Needles were inserted to depths of $1.5,0.8$, and $0.8 \mathrm{~cm}$ for pepper, tomato, and apple fruits, respectively. In rhubarb petioles and pokeweed stems, needles were inserted to penetrate the stem wall $(0.2$ to $0.3 \mathrm{~cm})$. For pepper fruits, the needle penetrated the fruit wall and extended into the large central gas cavity. For tomato and apple fruits, the needle was inserted and then cleaned by inserting a metal plunger through the needle to a depth several millimeters farther than the needle tip. For endive roots, it was necessary to first make a 1.2-cm-diameter hole into which a rubber serum cap was inserted and sealed. 
Additional gas-tight sealing was achieved by application of clear silicone rubber (Borden, Columbus, Ohio) on regions where the septa were attached to organ surfaces. In one experiment, the stem and stem scar regions of pepper and tomato fruit were sealed with silicone rubber to prevent water uptake. For rhubarb petioles and pokeweed stems, $6.0-\mathrm{cm}$ sections were cut and the ends were sealed with silicone rubber.

Manometers filled with an aqueous methylene blue solution were connected to all organs using $25-\mathrm{cm}$ lengths of $0.064-\mathrm{cm}$ (id.) flexible tubing. This method of measuring internal gas pressure-changes is a modification of the manometric techniques used previously to measure changes in internal gas pressure of brined cucumbers following carbonation or exchange with pure $\mathrm{O}_{2}$ (Corey et al., 1983a; Corey et al., 1983b). For temperature measurements in pepper fruits, a thermometer was implanted in the central gas cavity and sealed at the fruit surface. To maintain as constant a temperature as possible during the pressure measurements, temperatures of the water baths were monitored and adjusted by addition of small quantities of warmer or cooler water.

Water uptake. Uptake of acidified water from the baths by tomato and pepper fruits for the various temperature gradient treatments was monitored by recording the weight of individual fruits at various time intervals up to $22 \mathrm{hr}$.

\section{Results}

Partial pressure changes. Submergence of pepper fruits in acidified water $16 \mathrm{C}$ cooler than the initial temperature of the fruit resulted in a sharp decrease in internal gas pressure, which reached a maximum "change of about $34 \mathrm{~cm} \mathrm{H}_{2} \mathrm{O}$ after 15 min (Fig. 1). Changes in internal fruit temperature nearly paralleled changes in pressure. Fruit temperature approached the temperature of the water after 15 rein, indicating thermal equilibration of the fruit with the surrounding water bath. Changes in pressure were related linearly to changes in temperature with a slope of 2.-1 $\mathrm{cm} \mathrm{H}_{2} \mathrm{O} / \mathrm{C}$. Tomato fruits also developed internal partial vacuums to nearly the same extent as pepper fruits, except for

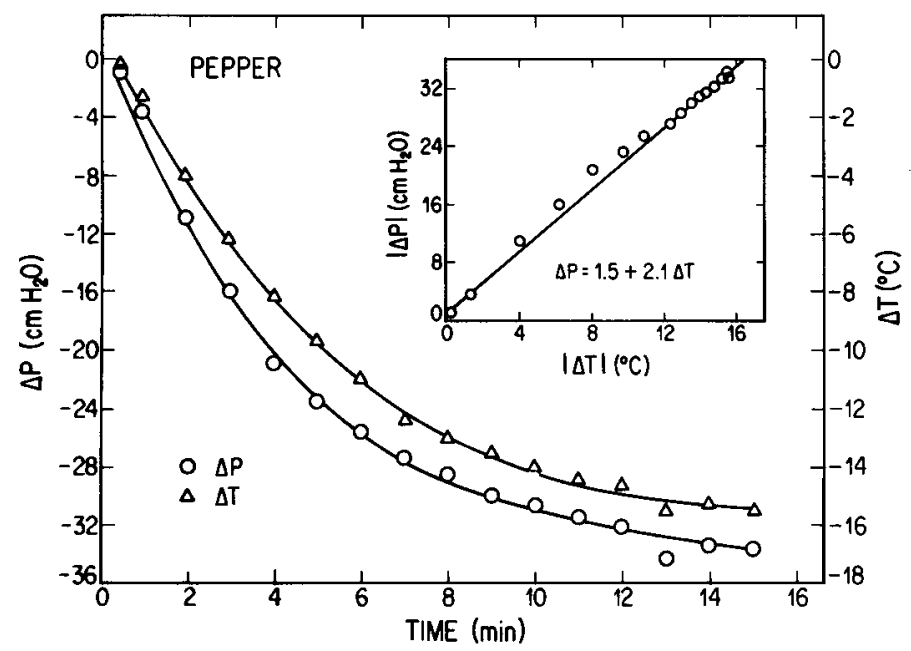

Fig. 1. Changes in internal gas pressure and temperature of pepper fruits following submergence in $8 \mathrm{C}$ acidified water $16 \mathrm{C}$ cooler than the initial temperature of the fruit $(24 \mathrm{C}) . \Delta \mathrm{P}$ denotes the difference in gas pressure between the fruit and atmosphere at the beginning of the experiment minus the difference at a given time. AT denotes the decline in temperature of the fruit with time. Points represent means of three replications. a small increase in pressure that occurred after $=14 \mathrm{~min}$ (Fig. 2).

Maximum changes in internal pressure $\left(\Delta \mathrm{P}_{\max }\right)$ increased with increasing difference in the initial temperature between the organ and the water ( $\Delta \mathrm{T} i)$ in both pepper and tomato fruit, regardless of whether the stem scar region was sealed. However, the $\Delta \mathrm{P}_{\text {max }}$ achieved was not linearly related to $\Delta \mathrm{T} \mathrm{i}$ (Fig. 3). Sealing the stem scar regions resulted in greater internal pressure changes than for fruit that were not sealed.

To determine if pressure increases would occur using temperature gradients in the opposite direction, pepper fruit were submerged in water $6 \mathrm{C}$ warmer than the initial temperature of the fruit. For four replications, the average $\Delta \mathrm{P}_{\text {max }}$ was $8.7 \pm$ $0.34 \mathrm{~cm} \mathrm{H}_{2} 0$. The time required to achieve $\Delta \mathrm{P}_{\text {max }}$ was $19.8 \pm$

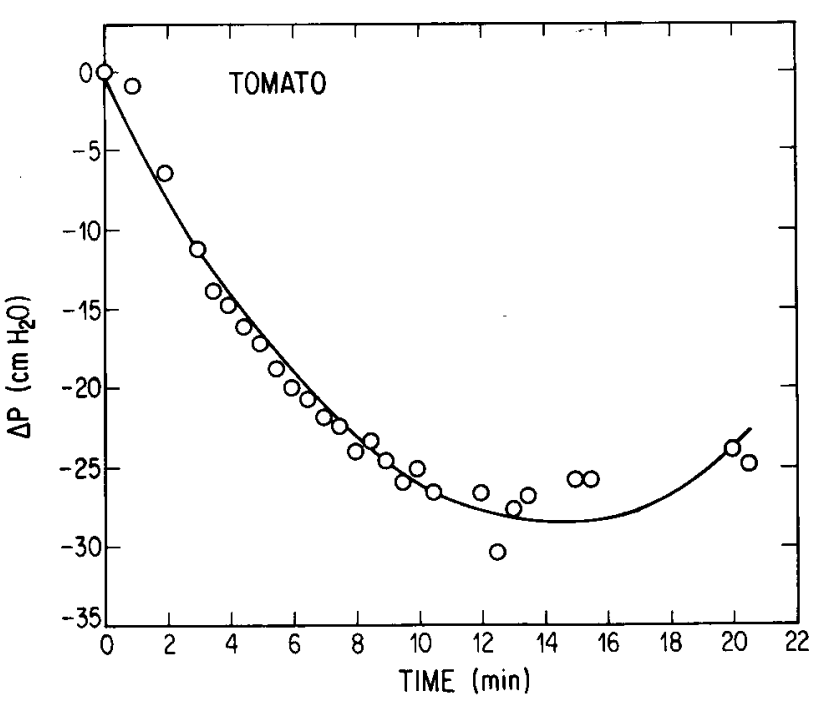

Fig. 2. Changes in internal gas pressure of mature-green tomato fruits following submergence in $8 \mathrm{C}$ acidified water $16 \mathrm{C}$ cooler than the initial temperature of the fruit $(24 \mathrm{C}) . \Delta \mathrm{P}$ denotes the difference in gas pressure between the fruit and atmosphere at the beginning of the experiment minus the difference at a given time. Points represent means of two replications.

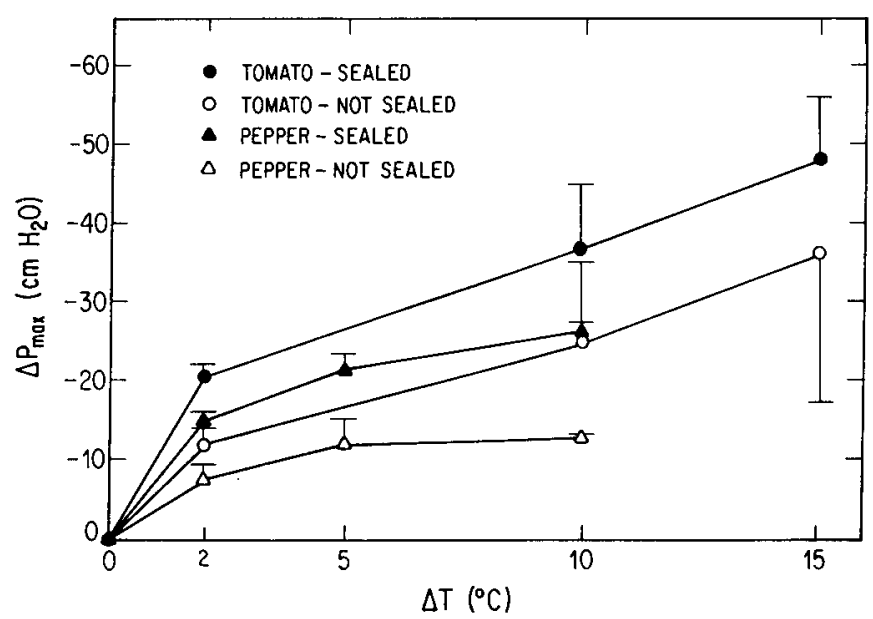

Fig. 3. Maximum changes in internal gas pressure in tomato and pepper fruits as affected by the initial difference in temperature between the fruit and water and by sealing of the stem scar region. Points represent means of two to four replications and vertical bars represent $1 \mathrm{SD}$. 
$6.3 \mathrm{~min}$. No pressure changes were detected when the temperatures of fruit and water bath were the same.

Various organs subjected to a temperature difference of SC in water developed $\Delta \mathrm{P}_{\text {max }}$ values that differed 5-fold (Table 1). Variability in the measurements for each organ was relatively low, as indicated by coefficients of variation ranging from $4.0 \%$ (endive root) to $22.9 \%$ (pokeweed stem). When no aqueous cooling medium was used and the organs were transferred to cold air $\left(\Delta \mathrm{T}_{\mathrm{i}}=15 \mathrm{C}\right)$, partial vacuums developed in pepper fruits, rhubarb petioles, and pokeweed stems, but not in apple fruits and endive roots (Table 1).

Water uptake. Water uptake by pepper fruits following submergence in water $17 \mathrm{C}$ cooler than the initial temperature of the fruits was rapid during the first hour and was followed by a nearly linear increase over the next $21 \mathrm{hr}$, reaching a maximum of $=17 \%$ of the initial fruit weight (Fig. 4). When fruits and water were at the same temperature, a slow linear increase in water uptake occurred, reaching a maximum of $<2 \%$. The same temperature treatments on tomato fruit resulted in similar trends for water uptake, but with a much lower maximum uptake (4\%) for $\Delta \mathrm{T} \mathrm{i}=17 \mathrm{C}$ and a slightly higher maximum uptake $(3 \%)$ for $\Delta \mathrm{T}_{\mathrm{i}}=0 \mathrm{C}$ (Fig. 5) than for pepper fruit (Fig. 4).

When pepper fruits were exposed to water baths having a warmer initial temperature than the fruit, water uptake was slow, and the rate was lower than that for fruits submerged in water of the same temperature (Fig. 6).

\section{Discussion}

Cooling of gases in a gas-tight, rigid system will result in a pressure decrease in accordance with the ideal gas law expressed as $\mathrm{PV}=\mathrm{nRT}$, where $\mathrm{P}$ is pressure, $\mathrm{V}$ is volume, $\mathrm{n}$ is number of moles, $\mathrm{R}$ is the molar gas constant, and $\mathrm{T}$ is temperature. If the number of moles of gas in the container is constant, the pressure of the gases in equilibrium with a given temperature change may be calculated according to $\mathrm{P}_{\mathrm{f}}=\mathrm{P}_{\mathrm{i}} \cdot \mathrm{T}_{\mathrm{r}} / \mathrm{T}_{\mathrm{i}}$, where the

Table 1. Maximum changes in gas pressure inside various plant organs following transfer to water $5 \mathrm{C}$ lower than the air temperature in which the organs were equilibrated.

\begin{tabular}{lc}
\hline \hline Organ & $\Delta \mathrm{P}_{\max }\left(\mathrm{cm} \mathrm{H}_{2} \mathrm{O}\right)^{\mathbf{z}}$ \\
\hline Tomato fruit & $-27.0 \pm 2.16$ \\
Pepper fruit & $-21.6 \pm 1.83$ \\
Apple fruit & $-31.4 \pm 3.17$ \\
Carrot root & $-36.3 \pm 3.51$ \\
Endive root & $-54.6 \pm 2.16$ \\
Rhubarb petiole & $-31.8 \pm 4.20$ \\
Pokeweed stem & $-11.4 \pm 2.61$ \\
\hline
\end{tabular}

${ }^{2}$ Values are relative to atmospheric pressure and represent means of three to four replications $\pm 1 \mathrm{SD}$.

Table 2. Maximum changes in gas pressure inside various plant organs following transfer to air $15 \mathrm{C}$ lower than the temperature of air in which the organs were equilibrated.

\begin{tabular}{lc}
\hline \hline Organ & $\Delta \mathrm{P}_{\max }\left(\mathrm{cm} \mathrm{H}_{2} \mathrm{O}\right)^{2}$ \\
\hline Pepper fruit & $-52.0 \pm 15.23$ \\
Apple fruit & 0 \\
Endive root & 0 \\
Rhubarb petiole & $-25.4 \pm 2.55$ \\
Pokeweed stem & $-35.7 \pm 4.04$ \\
\hline
\end{tabular}

'Values are relative to atmospheric pressure and represent means of three to four replications $\pm 1 \mathrm{SD}$.

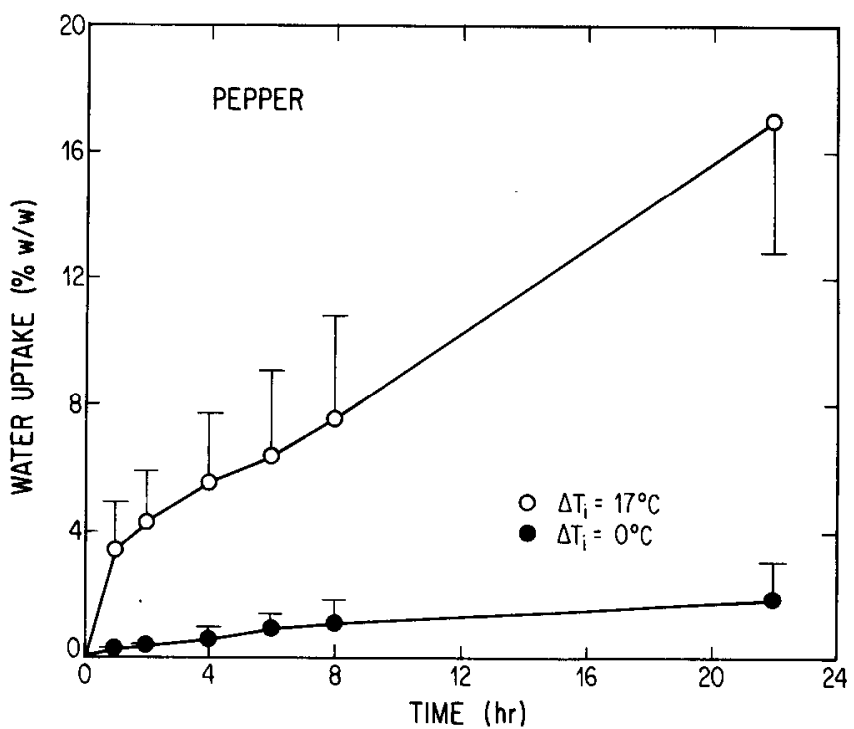

Fig. 4. Water uptake by whole pepper fruits following submergence in acidified water at the same temperature as the fruit $(\Delta \mathrm{T}=0 \mathrm{C})$ and in water $17 \mathrm{C}$ cooler than the fruit. Points represent means of four replications, and vertical bars represent 1 SD.

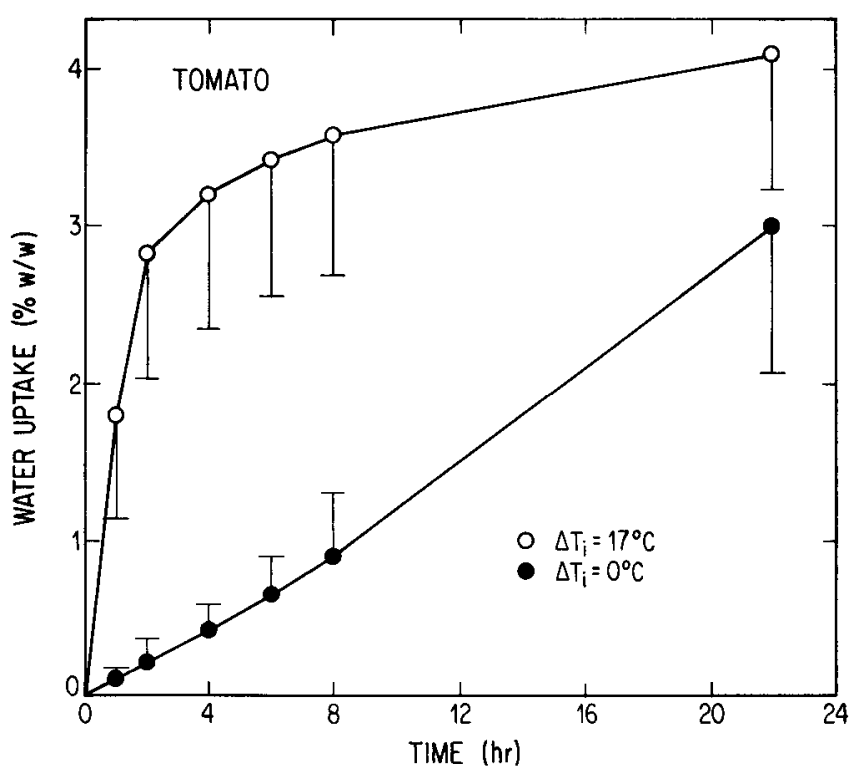

Fig. 5. Water uptake by whole mature-green tomato fruits following submergence in acidified water at the same temperature as the fruit $(\Delta \mathrm{T}=0 \mathrm{C}$ ) and in water $17 \mathrm{C}$ cooler than the fruit. Points represent means of four replications, and vertical bars represent $1 \mathrm{SD}$.

subscripts $\mathrm{i}$ and $\mathrm{f}$ denote initial and final, respectively. Within the range of ideal gas behavior, pressure changes are related linearly to temperature changes with a slope of $3.47 \mathrm{~cm} \mathrm{H}_{2} \mathrm{O}$ for every $1 \mathrm{C}$ change in temperature.

When plant organs with a relatively low permeability to gases or an additional diffusion barrier (e.g., water) are subjected to temperature changes, corresponding changes in gas pressure occur in the intercellular gas spaces of the tissue, but not in accordance with changes predicted by the ideal gas law. Pepper fruits exhibited pressure decreases of only $2.1 \mathrm{~cm} \mathrm{H}_{2} \mathrm{O}$ for every $1 \mathrm{C}$ change in temperature (Fig. 1). This deviation from ideal behavior may be attributed to concomitant pressure increases 


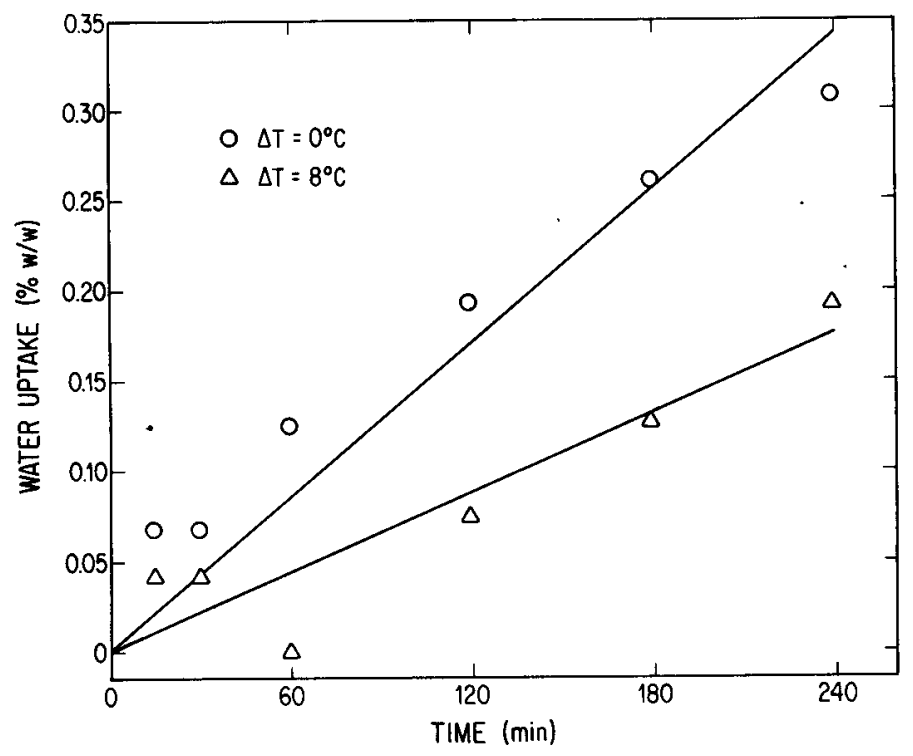

Fig. 6. Water uptake by whole pepper fruits following submergence in acidified water at the same temperature as the fruit $(\Delta \mathrm{T}-0 \mathrm{C})$ and in water $8 \mathrm{C}$ warmer than the fruit. Points represent means of six replications, and regression lines were forced through the origin.

due to water uptake. In the 15 min over which the experiment presented in Fig. 1 was conducted, pepper fruits took up an average of $1.170(\mathrm{w} / \mathrm{w})$ of water. Sealing fruit to prevent the uptake of water led to greater changes in gas pressure, more nearly approximating values predicted by the ideal gas law (Fig. $3)$.

Thermal equilibrium is attained rapidly in the water baths, so that sustained submergence enables the maintenance of a pressure gradient that drives the entrance of water (Figs. 4 and 5). Water uptake and internal transport are accelerated by the presence of temperature gradients. When no temperature gradient was present, the uptake of water for tomato and pepper fruits was slow, presumably taking place in response to a water potential gradient. In tomato fruits, a small increase in pressure was measured after $12 \mathrm{~min}$ exposure to a AT $=-16 \mathrm{C}$ (Fig. $2)$. Up to this time, and shortly thereafter, water uptake was rapid (Fig. 5) and would therefore explain the increase in pressure measured after $12 \mathrm{~min}$ (Fig. 2). After about $2 \mathrm{hr}$ in water, the rate of water uptake decreased rapidly, approaching a plateau as the intercellular spaces became liquid-filled. When pepper fruits were subjected to warmer water, the rate of water uptake was slower than the rate when there was no temperature gradient (Fig. 6). This may be attributed to the increased internal gas pressure causing an outward pressure gradient and suppressing water uptake. An explanation for only partial suppression of water uptake under these conditions was not investigated.

Thermal induction of total gas pressure gradients was a general phenomenon in plant organs (Tables 1 and 2). The extent of partial vacuum development, however, was variable, and, for organs submerged in water, probably depended on organ permeability to water. Maximum pressure changes in excess of predicted values often were measured, suggesting that another factor contributed to the development of internal partial vacuums. Consumption of $\mathrm{O}_{2}$ and evolution of $\mathrm{CO}_{2}$ in respiration lead to decreased and increased partial pressures, respectively, of these two gases. Changes in partial pressure of the two gases should offset one another and have no effect on the total internal pressure unless an additional sink for $\mathrm{CO}_{2}$ dissolution arises. Perhaps the infiltrated, acidified water blocked the escape of respiratory $\mathrm{CO}_{2}$ and further served as a reservoir for $\mathrm{CO}_{2}$ dissolution, leading to a lowered total gas pressure. This process presumably occurred when the infiltrated water equilibrated with the cellular fluids, since $\mathrm{CO}_{2}$ volubility increases with increasing $\mathrm{pH}$. Increased solubility of $\mathrm{CO}_{2}$ and other gases $\left(\mathrm{O}_{2}\right.$ and $\left.\mathrm{N}_{2}\right)$ would also be expected upon tissue cooling since gas volubility increases with decreasing temperature, thereby contributing partly to the higher-than-predicted partial vacuum.

The aqueous barrier to gas exchange was not necessary for development of partial vacuums. The partial vacuums measured in pepper fruits, rhubarb petioles, and pokeweed stems following exposure to cool air (Table 2) demonstrated this phenomenon. Apple fruits and endive roots did not develop partial vacuums in cool air, probably because the permeability of the tissue is sufficient to enable rapid gas transfer. These results suggest that tissues and organs most likely to develop partial vacuums in response to rapid temperature changes and a gas phase surface boundary are those with restricted permeability. Restricted permeability of bulky organs may arise from the absence of openings such as stomata and lenticils, a low frequency of openings, and closure, blockage, or nonfunctionality of openings.

The development of total pressure gradients in plant organs has several implications. With regard to postharvest handling of commodities, the obvious implications are in the use of water for washing, cooling, or providing dip treatments to produce. The use of water considerably cooler than the temperature of fruit will lead to the development of partial vacuums and water uptake. Water contaminated with microorganisms may lead to uptake of pathogens and lead to increased incidence of postharvest diseases (Bartz, 1982; Bartz and Showalter, 1981). Minimizing the temperature difference between commodity and the solution medium or decreasing the time of exposure would minimize the uptake of water and microorganisms. Results also suggest that air-cooling of organs with low gas permeability or organs with high gas permeability and packaged in perforated films may lead to the development of total gas pressure gradients and the transfer of gases by mass flow. This phenomenon may be desirable when produce is transferred from warm to cool environments, but undesirable when transfers to warmer environments are made.

There are other possibilities for the development of total gas pressure gradients within plant tissues. Diurnal changes in temperature leading to the development of gas pressure changes in intercellular spaces could provide a means for transport via mass flow of gases and solutions to and within attached organs. This mechanism may be widespread in plant tissues and contribute significantly to internal transport of substances. For example, internal mass flow of gases in plants maybe an important mechanism by which plant roots acquire sufficient $\mathrm{O}_{2}$ for respiration.

Evidence for thermally induced gas pressure gradients has been obtained for Nuphar lutea L. (Dacey, 1980) and for Nymphoides peltata (Grosse and Mevi-Schutz, 1987). Thomley (1987) suggested that the temperature-driven contribution to internal transport of solutions may be significant, but the work was restricted to a theoretical treatment of the phenomenon. Work needs to focus on the extent to which mass flow of gases may occur in plant organs and the role of thermally induced gas pressure gradients in the mass flow of solutions within plant tissues. 


\section{Literature Cited}

Bartz, Jerry A. 1982. Infiltration of tomatoes immersed at different temperatures to different depths in suspensions of Erwinia carotovora subsp. carotovora. Plant Dis. 66:302-306.

Bartz, Jerry A. and R.K. Showalter. 1981. Infiltration of tomatoes by aqueous bacterial suspensions. Phytopathology 71:515-518.

Ben-Yehoshua, S., S.P. Burg, and R. Young. 1985. Resistance of citrus fruit to mass transport of water vapor and other gases. Plant Physiol. 79:1048-1053.

Burg, S.P. and E.A. Burg. 1965. Gas exchange in fruits. Physiol. Plant. 18:870-884.

Cameron,.A.C. and S.F. Yang. 1982. A simple method for the determination of resistance to gas diffusion in plant organs. Plant Physiol. $70: 21-23$.
Corey, K. A., D.M. Pharr, and H.P. Fleming. 1983a. Pressure changes in oxygen-exchanged, brined cucumbers. J. Amer. Soc. Hort. Sci. 108:61-65.

Corey, K. A., D.M. Pharr, and H.P. Fleming. 1983b. Role of gas diffusion in bloater formation of pickling cucumbers. J. Food Sci. 48:389-393, 399.

Dacey, John W.H. 1980. Internal winds in water lilies: an adaptation for life in anaerobic sediments. Science 210:1017-1019.

Grosse, W. and J. Mevi-Schutz. 1987. A beneficial gas transport system in Nymphoides peltata. Amer. J. Bet. 74:947-952.

Solomos, T. 1987. Principles of gas exchange in bulky plant tissues. HortScience 22:766-771.

Thornley, J.H.M. 1987. The magnitude of the temperature-driven contribution to within-plant transport. Plant Cell Environ. 10:699-700. 